Am J Sports Med. 2016 December ; 44(12): 3146-3151. doi:10.1177/0363546516656373.

\title{
Utilization of ACL Injury Biomechanical and Neuromuscular Risk Profile Analysis to Determine the Effectiveness of Neuromuscular Training
}

\author{
Timothy E. Hewett, PhD ${ }^{\star}$, , Kevin R. Ford, PhD ${ }^{\ddagger}$, Yingying Y. Xu, MS§, Jane Khoury, PhD§, \\ and Gregory D. Myer, PhD§ \\ †Mayo Clinic, Rochester, Minnesota, USA \\ ‡High Point University, High Point, North Carolina, USA \\ $\S$ Cincinnati Children's Hospital Medical Center, Cincinnati, Ohio, USA
}

\section{Abstract}

Background-The widespread use of anterior cruciate ligament (ACL) injury prevention interventions has not been effective in reducing the injury incidence among female athletes who participate in high-risk sports.

Purpose/Hypothesis-The purpose of this study was to determine if biomechanical and neuromuscular factors that contribute to the knee abduction moment (KAM), a predictor of future ACL injuries, could be used to characterize athletes by a distinct factor. Specifically, we hypothesized that a priori selected biomechanical and neuromuscular factors would characterize participants into distinct at-risk profiles.

Study Design—Controlled laboratory study.

Methods-A total of 624 female athletes who participated in jumping, cutting, and pivoting sports underwent testing before their competitive season. During testing, athletes performed dropjump tasks from which biomechanical measures were captured. Using data from these tasks, latent profile analysis (LPA) was conducted to identify distinct profiles based on preintervention biomechanical and neuromuscular measures. As a validation, we examined whether the profile membership was a significant predictor of the KAM.

Results-LPA using 6 preintervention biomechanical measures selected a priori resulted in 3 distinct profiles, including a low (pro-file 1), moderate (profile 2), and high (profile 3) risk for

\footnotetext{
For reprints and permission queries, please visit SAGE's Web site at http://www.sagepub.com/journalsPermissions.nav. *Address correspondence to Timothy E. Hewett, PhD, Mayo Clinic, 200 First Street SW, Rochester, MN 55905, USA (hewett.timothy@mayo.edu).

Investigation performed at Mayo Clinic, Rochester, Minnesota, High Point University, High Point, North Carolina, and Cincinnati Children's Hospital Medical Center, Cincinnati, Ohio, USA

Presented at the 41st annual meeting of the AOSSM, Orlando, Florida, July 2015.

One or more of the authors has declared the following potential conflict of interest or source of funding: The authors acknowledge funding support from NFL Charities as well as from the National Institutes of Health/National Institute of Arthritis and Musculoskeletal and Skin Diseases (grants R01-AR055563, R01-AR056259, and R01-AR049735).
} 
ACL injuries. Athletes with profiles 2 and 3 had a significantly higher KAM compared with those with profile $1(P<.05)$.

Conclusion-This is the first study to use LPA of biomechanical landing data to create ACL injury risk profiles. Three distinct risk groups were identified based on differences in the peak KAM.

Clinical Relevance-These findings demonstrate the existence of discernable groups of athletes that may benefit from injury prevention interventions.

Study Registration-ClinicalTrials.gov NCT identifier: NCT01034527.

\section{Keywords}

anterior cruciate ligament; biomechanics; female athlete; neuromuscular training; risk profile

An anterior cruciate ligament (ACL) injury is one of the most common and debilitating knee injuries, especially among young female athletes. Seventy percent of ACL injuries are classified as noncontact, and more than $70 \%$ of those occur during jump landing. ${ }^{13}$ While noncontact injuries may be considered preventable, the role of biomechanical and neuromuscular risk factors in the prediction of ACL injuries remains controversial. External loads on the knee in the frontal plane, specifically the knee abduction moment (KAM), predict ACL injuries with high sensitivity and specificity. ${ }^{11}$ Faulty neuromuscular movement patterns that contribute to an increased KAM reportedly increase with age and first appear, most frequently in female patients, with the onset of pubertal maturation. ${ }^{10}$

Initial evidence indicated that neuromuscular training programs were effective in reducing the ACL injury risk in female athletes. ${ }^{9,15,19,23}$ However, even with widespread education and the institution of ACL injury prevention interventions, female athletes who participate in high-risk sports have not decreased their incidence of injuries. ${ }^{1,3,5,12}$ It has been suggested that the disparity between laboratory results and incidence outcomes may be related to the difficulty in implementing prevention programs. ${ }^{17}$ An alternative theory was proposed by Myklebust et al, ${ }^{20}$ who conducted a 10-year review of a Norwegian female handball ACL injury prevention study. The authors investigated whether ACL injury prevention initiatives had been effective. Myklebust et $\mathrm{al}^{20}$ reported that the ACL injury rate could be kept low through preventive initiatives but that risk factor studies are necessary to identify the patient's needs for special training and optimize the selection of preventive exercises.

Different athletic maneuvers may elicit specific movement patterns that are inherent to athletes at risk for ACL injuries. A double-leg landing immediately followed by a plyometric maximum vertical jump typically induces knee abduction motion in female athletes. Singleleg landings are also important tasks, as off-balance landings may mimic injurious conditions that are common during sports play. Consequently, 2 movements that may specifically play an important role in injury risk screening involve a drop vertical jump (DVJ), designed to monitor medial knee collapse, and a crossover single-leg drop (SCD), designed to elicit both knee abduction motion and trunk motion. Challenging athletes to perform these specific tasks during screening may help identify those athletes who will benefit most from training to reduce the risk of ACL injuries. 
Latent profile analysis (LPA) is a statistical tool that has primarily been used in the field of social science to characterize phenotypically alike patients into clusters or groups. The use of LPA is currently being explored more frequently in biomedical research. As such, LPA is a novel and potentially powerful tool to characterize the biomechanical and neuromuscular risk factors that underlie the ACL injury risk. The purpose of this article was to determine if LPA could be used to identify patient phenotypes based on the risk for future ACL injuries. Specifically, we hypothesized that biomechanical and neuromuscular factors contributing to the KAM during a DVJ and SCD would characterize participants into distinct injury risk profiles associated with a patient's risk for future ACL injuries.

\section{Methods}

\section{Participants}

This was a controlled laboratory study. The cohort consisted of 624 female athletes recruited from 52 regional basketball, soccer, and volleyball teams. Participants were required to have no history of ACL injuries or knee surgery and no lower extremity injuries or low back pain the year before study participation that required medical intervention. Testing was conducted before and after competitive seasons. Only data from the initial testing session, however, were used for the purpose of the current investigation. Participants and parents provided informed consent, approved by an institutional review board, before study procedures were initiated.

\section{Procedures}

Before initiating the screening process, participants' height and weight were recorded, and pubertal categories (prepubertal, pubertal, and postpubertal) were documented using the Pubertal Maturational Observational Scale (PMOS). ${ }^{4}$

All testing was conducted in a single laboratory. Three-dimensional (3D) motion analysis testing included the collection of kinematic data using a 10-camera motion capture system (Eagle Cameras; Motion Analysis Corp) at $240 \mathrm{~Hz}$. Kinetic data were collected at $1200 \mathrm{~Hz}$ from 2 force plates (AMTI) embedded into the floor and synchronized with motion data. Each participant was instrumented with 55 retroreflective markers, which were placed by a single experienced member of the investigative team. The methods and results for establishing marker placement reliability and validity, as well as a comprehensive description of marker locations, have been previously published. ${ }^{8,11}$ After marker placement, an astatic trial with the participant positioned in an anatomic neutral position was performed to define each patient's neutral alignment. Subsequent kinematic measures were referenced in relation to this position.

Testing consisted of the SCD, DVJ, and single-leg drop (SLD) maneuvers from a 31-cm box (Figure 1). For the purpose of developing injury risk profiles, only the SCD and DVJ tasks were utilized for analysis. The SCD was performed by balancing on 1 foot and then dropping forward and medially off the box, landing on the opposite leg. During the DVJ, the participant dropped off the box with both feet leaving at the same time, landing with each foot onto separate force platforms, and then immediately performing a maximum vertical 
jump. Tasks were performed in a randomized order with the landing side further randomized within each movement.

\section{Data Management}

The mean values for the 3 trials for each limb and each task were averaged, and the average was used for subsequent analysis. The interval of interest was the initial landing phase of each jump. Landing was defined from initial contact, when the vertical ground-reaction force first exceeded $10 \mathrm{~N}$, to the lowest point of the body's center of mass (COM). ${ }^{22}$ Marker trajectories and force plate data used for joint moment calculation were filtered with a bidirectional low-pass fourth-order Butterworth digital filter (12-Hz cutoff frequency). Force plate data used in calculating peak ground-reaction force data were additionally filtered with a bidirectional low-pass fourth-order Butterworth digital filter (100-Hz cutoff frequency). The hip joint center was determined using a validated anthropometrics-based calculation. ${ }^{2}$ Euler angles were used to describe lower extremity motions, and joint moments were calculated using inverse dynamics (C-Motion Inc; MathWorks Inc).

\section{Statistical Analysis}

Statistical testing was performed with SAS (version 9.3; SAS Institute). Descriptive analysis of all demographic data consisted of the calculation of means and SDs. Differences in demographic and biomechanical variables across groups were evaluated with analysis of variance. When significant differences were identified, post hoc testing was performed. Statistical significance was established at an alpha level of $P<.05$.

A cluster analytic technique, LPA, was used to examine whether participants could be grouped into distinct profiles based on a priori biomechanical characteristics during the tasks performed: ground-reaction force during the DVJ (GRF_DVJ), hip abduction moment during the SCD (HAdT_SCD), hip adduction moment maximum during the SCD (HipADD_SCD), hip adduction moment minimum during the SCD (HipM_SCD), hip adduction moment minimum during the DVJ (HipM_DVJ), and peak frontal-plane pelvis angle during the SCD (PelvisAng_SCD). LPA was implemented in Mplus version 5. ${ }^{16}$ The implementation of LPA was carried out in a stepwise fashion beginning with 2 profiles and then the number of profiles increasing by 1 at each step. Models with a successively increased number of profiles were compared using the Bayesian information criterion (BIC) and adjusted for sample size. A lower BIC indicated a better model fit. The number of profiles was deemed adequate when no significant drop in the BIC was seen when the number of profiles was increased.

Other criteria to guide the decision on the optimal number of profiles include the LoMendell-Rubin adjusted likelihood ratio test, ${ }^{14}$ average posterior probability, entropy, and profile membership probabilities. The correlation between variables was accounted for in the analysis including hip adduction with both ground-reaction force and hip abduction moment. The validation of profile groups involved an examination of each group as an independent predictor of the KAM. 


\section{Results}

Among the initial cohort, 66 individuals did not make the team roster, posttest data only was available for 18 individuals, and 73 individuals were excluded from the analysis secondary to incomplete or bad data. This left 467 athletes whose data were available for analysis. As a group, the participants had a mean $( \pm \mathrm{SD})$ age of $14 \pm 1.8$ years, mean height of $160 \pm 8.1$ $\mathrm{cm}$, mean weight of $55 \pm 12 \mathrm{~kg}$, and mean body mass index (BMI) of $21.2 \pm 3.6 \mathrm{~kg} / \mathrm{m}^{2}$. Maturation classification included $50.8 \%$ of the participants classified as pubertal stage, $43.6 \%$ as postpubertal stage, and $5.6 \%$ as prepubertal stage. The majority of the athletes participated in basketball (48.8\%), followed by volleyball (26.6\%) and soccer (24.6\%). The percentages of athletes from high schools versus middle schools were approximately the same: $49.0 \%$ versus $51.0 \%$, respectively.

LPA resulted in 3 distinct risk profiles, which were defined as a low (profile 1), moderate (profile 2), and high (profile 3) risk for future ACL injuries. The profiles exhibited significant differences in age, pubertal stage, weight, height, and BMI $(P<.05)$ (Table 1). Adjusted and unadjusted KAMs were significantly different during the DVJ between profiles 1 and 2 and between profiles 1 and $3(P<.05)$ (Table 1). Adjusted and unadjusted KAMs were significantly different during the SCD across all profiles $(P<.05)$ (Table 1$)$.

There were differences across groups for the biomechanical variables used to develop the risk profiles. There were significant differences across all groups for GRF_DVJ, HAdT_SCD, and HipADD_SCD $(P<.05)$ (Table 2$)$. There were significant differences between profiles 1 and 2 and between profiles 1 and 3 for HipM_DVJ $(P<.05)$ (Table 2). There were also significant differences between profiles 1 and 3 and between profiles 2 and 3 for PelvisAng_SCD $(P<.05)$ (Table 2$)$.

\section{Discussion}

To our knowledge, this is the first study to use LPA to identify distinct profile groups of athletes using biomechanical landing data. This included $14 \%$ of the cohort classified as high risk, $72 \%$ as moderate risk, and $14 \%$ as low risk for future ACL injuries. LPA provided group separation using multiple combined movement analyses, and 3 distinct profiles were observed using peak trunk and hip biomechanical measures. Group differences in the peak KAM during the DVJ across the profiles were significant (profile $2>$ profile 1 and profile 3 $>$ profile 1), whether unadjusted or adjusted for age and pubertal stage. Profile 1 was more likely to consist of younger and prepubertal athletes, while profile 3 was more likely to consist of older and postpubertal athletes.

Three distinct groups were identified with potentially disparate risks for ACL injuries. These results suggest that injury prevention strategies could be applied to female athletes who are at greatest risk for future injuries. Previously, Myer et al ${ }^{17}$ evaluated kinematics and kinetics during a DVJ test in 18 uninjured high school female athletes before and after a 7-week neuromuscular training program. Prediction of the ACL injury risk was calculated using the external KAM, with high risk defined as values exceeding $25.25 \mathrm{~N} \cdot \mathrm{m}$ and low risk defined as values below this point. Myer et al ${ }^{17}$ reported that athletes classified as high risk exhibited 
a decrease in their KAMs by $13 \%$ after training while low-risk athletes did not demonstrate any change in abduction moments. The authors also reported, however, that high-risk athletes who participated in neuro-muscular training did not reduce their KAMs to levels similar to the low-risk group. ${ }^{17}$ This prompted Myer et al ${ }^{17}$ to suggest that alterations in current neuromuscular training programs may be necessary for high-risk athletes to substantially decrease their ACL injury risk. The work of Myer et al ${ }^{17}$ was limited by the simple delineation of the future injury risk by using a single frontal-plane knee moment value to distinguish athlete groups. In the current investigation, a far more robust and sophisticated approach using LPA of several biomechanical movement characteristics was implemented to identify injury risk groups. This included trunk and hip biomechanics that are known to contribute to frontal-plane knee loads. The validation of low, moderate, and high injury risk groups was established by evaluating differences in frontal-plane knee loading. Results from the current investigation provide evidence to guide the selective implementation of prevention programs among athletes who are at greatest risk for future injuries as an effective utilization of time and resources.

For those patients who are classified as low risk, programs implemented as part of a team warm-up ${ }^{21}$ may be adequate. Alternatively, patients who are moderate or high risk may require supervised training with structured verbal and visual feedback ${ }^{18}$ as well as training that includes an emphasis on core and hip control. ${ }^{6}$ The effects of different types of ACL injury prevention programs across risk groups must, however, be assessed with future studies.

Neuromuscular deficits associated with an increased KAM appear to increase with age, and their increased prevalence largely coincides with the onset of maturation. ${ }^{10}$ Neuromuscular deficits associated with an increased KAM appear to increase with age, and their increased prevalence largely coincides with the onset of maturation. This investigation identified significant group differences in the peak KAM across profiles. Previous studies by Hewett et $\mathrm{al}^{10}$ and Ford et al ${ }^{7}$ demonstrated that the KAM increases with maturation. A high KAM is considered a significant marker of the risk for future injuries, as patients who later sustain an ACL injury have prospectively exhibited large frontal-plane knee loads. ${ }^{11}$ Further investigation is required to prospectively study the effects of each of these group profiles on the KAM and the ACL injury risk in both groups and individual patients.

In a laboratory setting, it can be challenging to both elicit and quantify the effects of the proximal influence on lower extremity stability. To overcome this challenge, the current study employed movement tasks that were designed to perturb both the knee and trunk. The double-leg DVJ is the most commonly reported task in the literature to assess dynamic lower extremity biomechanics in an athletic population. The task requires participants to drop off a $31 \mathrm{~cm}$-high box with both feet at the same time and, on landing, immediately perform a bilateral vertical jump with maximal effort. This center line movement largely ensures that the COM remains medial to both knee joint centers throughout the maneuver and typically does not elicit movement of the COM in the frontal plane relative to the knee joints. The task does, however, permit movement of the knees relative to the plantar surface of the feet. Hence, this movement elicits its primary perturbation to the knee bilaterally. The second task, the SCD, simultaneously requires synergistic control of both the knee and trunk. This 
task consists of a lateral hop from a box in which the participant lands on the limb opposite the initial stance leg. Lateral momentum of the COM during the SCD elicits trunk perturbation on landing and simultaneously challenges the lower extremity and hip musculature to maintain alignment of the knee over the stance foot. Hence, this movement elicits its primary perturbation to the bilateral knee joints and the trunk simultaneously. The third task, an SLD landing from a box, did not contribute to LPA for group development. The SLD landing permits movement of the trunk and pelvis but does not necessarily elicit these factors. Athletes must maintain alignment of their knee over their foot to successfully perform this task. Thus, the challenge to the lower extremity musculature makes this task particularly useful for identifying and eliciting asymmetries, particularly as measures of functional progress after injuries and ACL reconstruction. Hence, the SLD movement elicits its primary perturbation to the trunk only.

This study is not without limitations. This study established a biomechanical and neuromuscular rationale for distinguishing between athlete groups based on their risk for future ACL injuries. Validation of risk group profiles, however, will be necessary with a clinical trial. This study only assessed young female athletes. Furthermore, the effectiveness of a differential injury prevention approach based on the risk profile in the effects on reducing injury rates must also be assessed with a clinical trial. While it is well documented that female athletes who participate in jumping, cutting, and pivoting sports are at high risk for future ACL injuries, male athletes at risk for injuries may necessitate a distinct classification algorithm. This study utilized sophisticated methods to collect 3D biomechanical and neuromuscular characteristics for the development of risk groups. A correlate of the screening examination that is easy to administer and does not require expensive equipment will be necessary to translate the classification algorithm into clinical practice.

\section{Conclusion}

The findings of the current study suggest an alternative approach to ACL injury risk screening. The clustering of biomechanical and neuromuscular risk factors associated with an increased KAM and potential increased risk for ACL injuries indicates that there are select groupings of young female athletes who may benefit the most from preventive core stability-based intervention protocols. The findings also demonstrate that those young athletes undergoing and completing pubertal maturation are most likely to benefit from this training.

\section{Acknowledgments}

The authors acknowledge the Sports Medicine Biodynamics Team, who worked together to make large data collection sessions possible. The authors also thank Boone County schools for their invaluable support to complete this project.

\section{References}

1. Agel J, Arendt EA, Bershadsky B. Anterior cruciate ligament injury in National Collegiate Athletic Association basketball and soccer: a 13-year review. Am J Sports Med. 2005; 33(4):524-530. [PubMed: 15722283] 
2. Bell AL, Pedersen DR, Brand RA. A comparison of the accuracy of several hip center location prediction methods. J Biomech. 1990; 23(6):617-621. [PubMed: 2341423]

3. Brophy RH, Stepan JG, Silvers HJ, Mandelbaum BR. Defending puts the anterior cruciate ligament at risk during soccer: a gender-based analysis. Sports Health. 2015; 7(3):244-249. [PubMed: 26131302]

4. Davies PL, Rose JD. Motor skills of typically developing adolescents: awkwardness or improvement? Phys Occup Ther Pediatr. 2000; 20(1):19-42. [PubMed: 11293913]

5. Deitch JR, Starkey C, Walters SL, Moseley JB. Injury risk in professional basketball players: a comparison of Women's National Basketball Association and National Basketball Association athletes. Am J Sports Med. 2006; 34(7):1077-1083. [PubMed: 16493173]

6. Di Stasi S, Myer GD, Hewett TE. Neuromuscular training to target deficits associated with second anterior cruciate ligament injury. J Orthop Sports Phys Ther. 2013; 43(11):777-792, A1-A11. [PubMed: 24175599]

7. Ford KR, Myer GD, Hewett TE. Longitudinal effects of maturation on lower extremity joint stiffness in adolescent athletes. Am J Sports Med. 2010; 38(9):1829-1837. [PubMed: 20522830]

8. Ford KR, Myer GD, Hewett TE. Reliability of landing 3D motion analysis: implications for longitudinal analyses. Med Sci Sports Exerc. 2007; 39(11):2021-2028. [PubMed: 17986911]

9. Hewett TE, Lindenfeld TN, Riccobene JV, Noyes FR. The effect of neuromuscular training on the incidence of knee injury in female athletes: a prospective study. Am J Sports Med. 1999; 27(6):699706. [PubMed: 10569353]

10. Hewett TE, Myer GD, Ford KR. Decrease in neuromuscular control about the knee with maturation in female athletes. J Bone Joint Surg Am. 2004; 86(8):1601-1608. [PubMed: 15292405]

11. Hewett TE, Myer GD, Ford KR, et al. Biomechanical measures of neuromuscular control and valgus loading of the knee predict anterior cruciate ligament injury risk in female athletes: a prospective study. Am J Sports Med. 2005; 33(4):492-501. [PubMed: 15722287]

12. Joseph AM, Collins CL, Henke NM, Yard EE, Fields SK, Comstock RD. A multisport epidemiologic comparison of anterior cruciate ligament injuries in high school athletics. $\mathrm{J}$ Athl Train. 2013; 48(6):810-817. [PubMed: 24143905]

13. Kim S, Bosque J, Meehan JP, Jamali A, Marder R. Increase in outpatient knee arthroscopy in the United States: a comparison of National Surveys of Ambulatory Surgery, 1996 and 2006. J Bone Joint Surg Am. 2011; 93(11):994-1000. [PubMed: 21531866]

14. Lo Y, Mendell NR, Rubin DB. Testing the number of components in a normal mixture. Biometrika. 2001; 88:767-778.

15. Mandelbaum BR, Silvers HJ, Watanabe DS, et al. Effectiveness of a neuromuscular and proprioceptive training program in preventing anterior cruciate ligament injuries in female athletes: 2-year follow-up. Am J Sports Med. 2005; 33(7):1003-1010. [PubMed: 15888716]

16. Muthen, LK., Muthen, BO. Mplus User's Guide: Version 5. Los Angeles: Muthen and Muthen; 2007.

17. Myer GD, Ford KR, Brent JL, Hewett TE. Differential neuromuscular training effects on ACL injury risk factors in "high-risk" versus "low-risk" athletes. BMC Musculoskel Dis. 2007; 8:39.

18. Myer GD, Stroube BW, DiCesare CA, et al. Augmented feedback supports skill transfer and reduces high-risk injury landing mechanics: a double-blind, randomized controlled laboratory study. Am J Sports Med. 2013; 41(3):669-677. [PubMed: 23371471]

19. Myklebust G, Engebretsen L, Braekken IH, Skjolberg A, Olsen OE, Bahr R. Prevention of anterior cruciate ligament injuries in female team handball players: a prospective intervention study over three seasons. Clin J Sport Med. 2003; 13(2):71-78. [PubMed: 12629423]

20. Myklebust G, Skjolberg A, Bahr R. ACL injury incidence in female handball 10 years after the Norwegian ACL prevention study: important lessons learned. Br J Sports Med. 2013; 47(8):476479. [PubMed: 23403528]

21. Olsen OE, Myklebust G, Engebretsen L, Holme I, Bahr R. Exercises to prevent lower limb injuries in youth sports: cluster randomised controlled trial. BMJ. 2005; 330(7489):449. [PubMed: 15699058] 
22. Paterno MV, Schmitt LC, Ford KR, et al. Biomechanical measures during landing and postural stability predict second anterior cruciate ligament injury after anterior cruciate ligament reconstruction and return to sport. Am J Sports Med. 2010; 38(10):1968-1978. [PubMed: 20702858]

23. Petersen W, Braun C, Bock W, et al. A controlled prospective case control study of a prevention training program in female team hand-ball players: the German experience. Arch Orthop Trauma Surg. 2006; 125(9):614-621. 

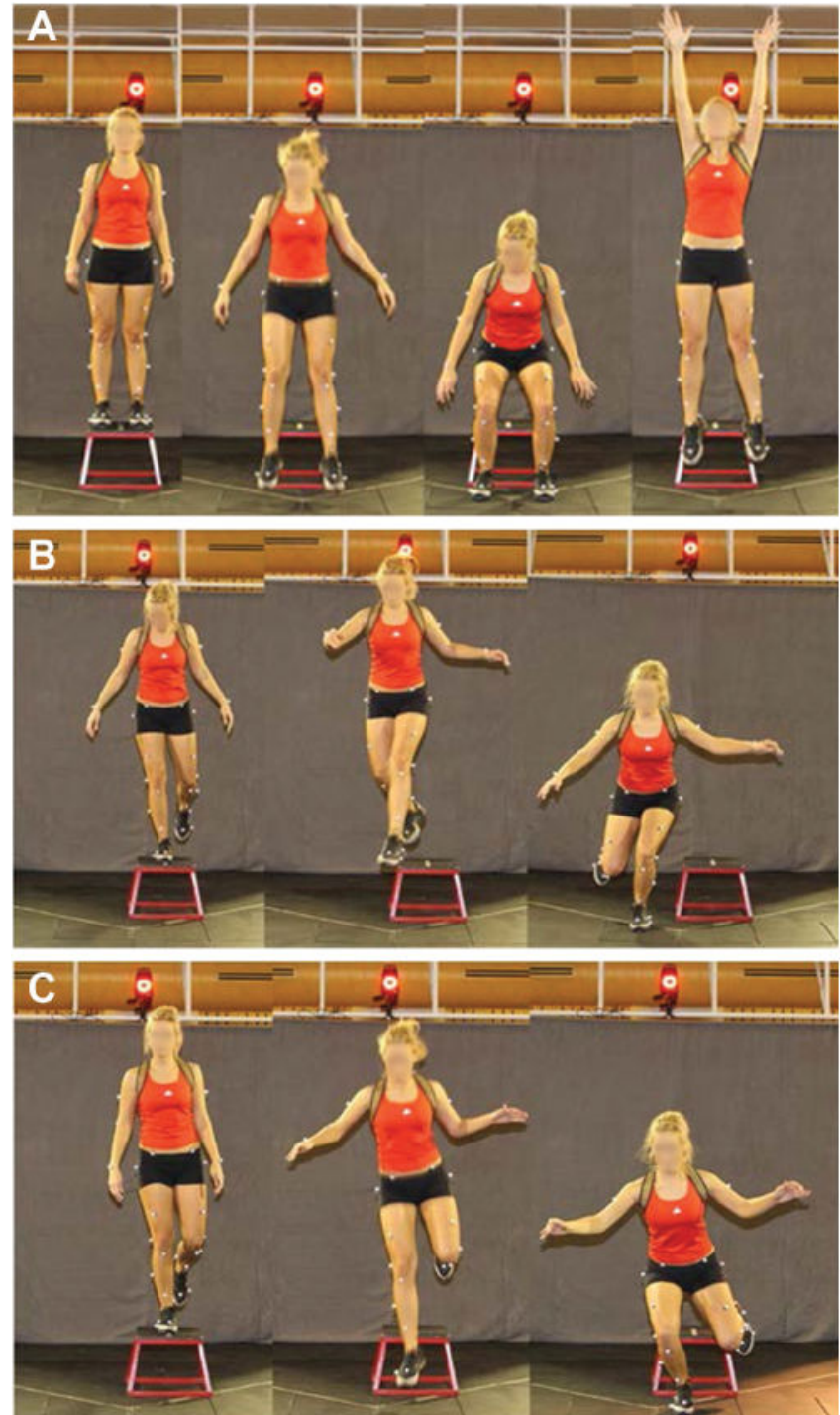

Figure 1. The 3 testing maneuvers: (A) drop vertical jump, (B) crossover single-leg drop, and (C) single-leg drop 
Table 1

Demographic Characteristics and Preintervention Demographic, Anthropometric, and Biomechanical Measures of Athletes by LPA Risk Profile ${ }^{a}$

\begin{tabular}{|c|c|c|c|}
\hline & Profile $1($ Low; $n=62)$ & Profile 2 (Moderate; $\mathbf{n}=\mathbf{3 3 7}$ ) & Profile 3 (High; $n=68$ ) \\
\hline Age, $, b, c, d$ y & $12.6 \pm 0.2$ & $14.1 \pm 0.1$ & $14.9 \pm 0.2$ \\
\hline \multicolumn{4}{|l|}{ Pubertal stage, n (\%) } \\
\hline Prepubertal & $12(19)$ & $14(4)$ & $0(0)$ \\
\hline Pubertal & $40(65)$ & $175(52)$ & $21(32)$ \\
\hline Postpubertal & $10(16)$ & $148(44)$ & $45(68)$ \\
\hline Weight, $b, c, d \mathrm{~kg}$ & $41.2 \pm 1.2$ & $54.5 \pm 0.5$ & $70.1 \pm 1.1$ \\
\hline Height, cm & $151.2 \pm 0.9$ & $161.1 \pm 0.4$ & $166.7 \pm 0.8$ \\
\hline BMI, $\mathrm{kg} / \mathrm{m}^{2}$ & $17.9 \pm 0.4$ & $20.9 \pm 0.2$ & $25.2 \pm 0.4$ \\
\hline \multicolumn{4}{|l|}{ KAM (DVJ), Nm } \\
\hline Unadjusted $b, c$ & $-15.3 \pm 1.5$ & $-23.2 \pm 0.6$ & $-25.6 \pm 1.4$ \\
\hline Adjusted for age and pubertal stage $b, c$ & $-15.6 \pm 1.6$ & $-22.4 \pm 1.0$ & $-24.8 \pm 1.7$ \\
\hline \multicolumn{4}{|l|}{ KAM (SCD), Nm } \\
\hline Unadjusted $^{b, c, d}$ & $-8.1 \pm 1.6$ & $-12.4 \pm 0.7$ & $-16.1 \pm 1.6$ \\
\hline Adjusted for age and pubertal stage $b, c, d$ & $-4.6 \pm 1.8$ & $-11.3 \pm 1.0$ & $-15.9 \pm 1.8$ \\
\hline \multirow{2}{*}{\multicolumn{4}{|c|}{$\begin{array}{l}\text { Data are reported as least squares mean } \pm \text { standard error unless otherwise indicated. BMI, body mass index; DVJ, drop vertical jump; } \\
\text { bduction moment; LPA, latent profile analysis; SCD, crossover single-leg drop. }\end{array}$}} \\
\hline & & & \\
\hline \multicolumn{4}{|c|}{ Statistically significant difference between profiles 1 and $3(P .05)$. } \\
\hline
\end{tabular}


Table 2

\section{Results for Preintervention Biomechanical Variables by LPA Risk Profile ${ }^{a}$}

\begin{tabular}{lccc}
\hline Biomechanical Variable & Profile $\mathbf{1}($ Low; n = 62) & Profile 2 (Moderate; n = 337) & Profile 3 (High; n = 68) \\
\hline GRF_DVJ $b, c, d$ & $701.3 \pm 33.4$ & $1035.1 \pm 14.4$ & $1376.8 \pm 32.2$ \\
HAdT_SCD $b, c, d$ & $19.3 \pm 1.0$ & $27.6 \pm 0.4$ & $44.2 \pm 1.0$ \\
HipADD_SCD $b, c, d$ & $62.9 \pm 2.8$ & $94.4 \pm 1.2$ & $146.1 \pm 2.7$ \\
HipM_SCD & $-5.6 \pm 1.7$ & $-4.7 \pm 0.7$ & $-5.0 \pm 1.6$ \\
HipM_DVJ $b, c$ & $-14.5 \pm 1.5$ & $-21.0 \pm 0.6$ & $-24.0 \pm 1.4$ \\
PelvisAng_SCD ${ }^{c}$ & $9.1 \pm 0.4$ & $9.3 \pm 0.2$ & $10.8 \pm 0.4$ \\
\hline
\end{tabular}

${ }^{a}$ Data are reported as mean \pm standard error. GRF_DVJ, ground-reaction force during the drop vertical jump; HAdT_SCD, hip abduction moment during the crossover single-leg drop; HipADD_SCD, hip adduction moment maximum during the crossover single-leg drop; HipM_DVJ, hip adduction moment minimum during the drop vertical jump; HipM_SCD, hip adduction moment minimum during the crossover single-leg drop; LPA, latent profile analysis; PelvisAng_SCD, peak frontal-plane pelvis angle during the crossover single-leg drop.

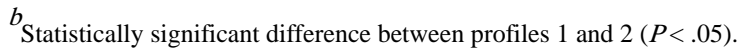

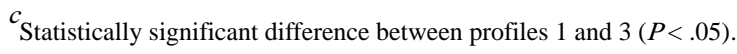

${ }^{d}$ Statistically significant difference between profiles 2 and $3(P<.05)$. 